\title{
ON THE NUMBER OF PRIME FACTORS OF AN ODD PERFECT NUMBER
}

\author{
PASCAL OCHEM AND MICHAËL RAO
}

\begin{abstract}
Let $\Omega(n)$ and $\omega(n)$ denote, respectively, the total number of prime factors and the number of distinct prime factors of the integer $n$. Euler proved that an odd perfect number $N$ is of the form $N=p^{e} m^{2}$ where $p \equiv e \equiv 1$ $(\bmod 4), p$ is prime, and $p \nmid m$. This implies that $\Omega(N) \geq 2 \omega(N)-1$. We prove that $\Omega(N) \geq(18 \omega(N)-31) / 7$ and $\Omega(N) \geq 2 \omega(N)+51$.
\end{abstract}

\section{INTRODUCTION}

A natural number $N$ is said to be perfect if it is equal to the sum of its positive divisors (excluding $N$ ). It is well known that an even natural number $N$ is perfect if and only if $N=2^{k-1}\left(2^{k}-1\right)$ for an integer $k$ such that $2^{k}-1$ is a Mersenne prime. On the other hand, it is a long-standing open question whether an odd perfect number exists.

In order to investigate this question, several authors gave necessary conditions for the existence of an odd perfect number $N$. Let $\Omega(n)$ and $\omega(n)$ denote, respectively, the total number of prime factors and the number of distinct prime factors of the integer $n$. Euler proved that $N=p^{e} m^{2}$ for a prime $p$, with $p \equiv e \equiv 1(\bmod 4)$, $p$ is prime, and $p \nmid m$. Moreover, recent results showed that $N>10^{1500}$ [4, $\omega(N) \geq 9[3$, and $\Omega(N) \geq 101[4$.

In this paper, we study the relationship between $\Omega(N)$ and $\omega(N)$. By Euler's result, we have $\Omega(N) \geq 2 \omega(N)-1$. Steuerwald [6] proved that $m$ is not squarefree, that is, the exponents of the non-special primes cannot be all equal to 2 . This implies that $\Omega(N) \geq 2 \omega(N)+1$. We improve this inequality in two ways:

Theorem 1. If $N$ is an odd perfect number, then $\Omega(N) \geq(18 \omega(N)-31) / 7$.

Theorem 2. If $N$ is an odd perfect number, then $\Omega(N) \geq 2 \omega(N)+51$.

We prove Theorem 1 in Section 3 using standard arguments. We prove Theorem 2 in Section 4 via computations using the general method in [4].

To summarize the known results for $\Omega(N)$, we have

$$
\Omega(N) \geq \max \{101,2 \omega(N)+51,(18 \omega(N)-31) / 7\} .
$$

\section{Preliminaries}

Let $n$ be a natural number. Let $\sigma(n)$ denote the sum of the positive divisors of $n$, and let $\sigma_{-1}(n)=\frac{\sigma(n)}{n}$ be the abundancy of $n$. Clearly, $n$ is perfect if and only if $\sigma_{-1}(n)=2$. We first recall some easy results on the functions $\sigma$ and $\sigma_{-1}$. If $p$ is

Received by the editor September 15, 2012 and, in revised form, December 18, 2012. 2010 Mathematics Subject Classification. Primary 11A25, 11A51. 
prime, $\sigma\left(p^{q}\right)=\frac{p^{q+1}-1}{p-1}$, and $\sigma_{-1}\left(p^{\infty}\right)=\lim _{q \rightarrow+\infty} \sigma_{-1}\left(p^{q}\right)=\frac{p}{p-1}$. If $\operatorname{gcd}(a, b)=1$, then $\sigma(a b)=\sigma(a) \sigma(b)$ and $\sigma_{-1}(a b)=\sigma_{-1}(a) \sigma_{-1}(b)$.

Euler proved that if an odd perfect number $N$ exists, then it is of the form $N=p^{e} m^{2}$ where $p \equiv e \equiv 1(\bmod 4), p$ is prime, and $p \nmid m$. The prime $p$ is said to be the special prime.

\section{Proof of $\Omega(N) \geq(18 \omega(N)-31) / 7$}

We want to obtain a result of the form $\Omega(N) \geq a \omega(N)-c$ for some $a>2$ using the following idea. If $a$ is close to 2 , then $N$ has a large number of prime factors $p$ such that both $p^{2} \| N$ and $p \| \sigma\left(q^{2}\right)$ where $q^{2} \| N$. It is well known (see [5]) that for primes $t, r$, and $s$ such that $t \mid \sigma\left(r^{s-1}\right)$, either $t=s$ or $t \equiv 1 \bmod s$. In particular, this gives $p \equiv 1 \bmod 3$ and thus $3 \mid \sigma\left(p^{2}\right)$. The exponent of the prime 3 is then large, so that $\Omega(N)$ is significantly greater than $2 \omega(N)$.

Now we detail the number of certain types of factors of $N$ and obtain the results by contradiction with the involved quantities.

- $p=\omega(N)$ : number of distinct prime factors,

- $f=\Omega(N)$ : total number of prime factors,

- $p_{2}$ : number of distinct prime factors with exponent 2 , distinct from 3 ,

- $p_{2,1}$ : number of distinct prime factors with exponent 2 congruent to $1 \mathrm{mod}$ 3,

- $p_{4}$ : number of distinct prime factors with exponent at least 4 , distinct from 3 and the special prime,

- $f_{4}$ : total number of prime factors with exponent at least 4 , distinct from 3 and the special prime,

- $e$ : exponent of the special prime,

- $f_{3}$ : exponent of the prime 3 .

Now we obtain useful inequalities among these quantities. The special exponent is at least 1 :

$$
1 \leq e
$$

By detailing the total number of prime factors, we have

$$
e+f_{3}+2 p_{2}+f_{4}=f .
$$

By considering the prime factors (distinct from 3 and the special prime) with exponent at least 4 , we have

$$
4 p_{4} \leq f_{4} .
$$

As already mentioned, if $p \equiv 1 \bmod 3$ and $p^{2} \| N$, then $3 \mid \sigma\left(p^{2}\right)$, so that

$$
p_{2,1} \leq f_{3} .
$$

Let us consider the number of distinct prime factors. We have the special prime, the primes from $p_{2}$ and $p_{4}$, and maybe the prime 3 . So it is $1+p_{2}+p_{4}$ if $f_{3}=0$ and $2+p_{2}+p_{4}$ if $f_{3} \geq 2$. Thus, we have

$$
p \leq f_{3} / 2+1+p_{2}+p_{4}
$$

and

$$
p \leq 2+p_{2}+p_{4}
$$


For the sake of contradiction, we suppose that

$$
7 f \leq 18 p-32 \text {. }
$$

The following lemma is useful to obtain one last inequality:

Lemma 3. Let $p$, $q$, and $r$ be positive integers. If $p^{2}+p+1=r$ and $q^{2}+q+1=3 r$, then $p$ is not an odd prime.

Proof. Since $q^{2}+q+1 \equiv 0 \bmod 3$, then $q \equiv 1 \bmod 3$ and we set $q=3 s+1$. The equality $q^{2}+q+1=3\left(p^{2}+p+1\right)$ reduces to $3 s(s+1)=p(p+1)$. Notice that $p$ divides $3 s(s+1)$, so that if $p$ is an odd prime, then either $p|3, p| s$, or $p \mid(s+1)$. We have $p=3$ in the first case, which gives no solution. We have $s \geq p-1$ in the other two cases, so that $p(p+1)=3 s(s+1) \geq 3(p-1) p$. This gives $p+1 \geq 3(p-1)$, so that $p \leq 2$, which is a contradiction.

Let $K$ be the multiset of all the primes distinct from 3 produced by all the components $\sigma\left(p^{2}\right)$ of $N$. The primes in $K$ are $1 \bmod 3$, so $|K| \leq e+2 p_{2,1}+f_{4}$. For a prime $u>3$, let $\alpha(u)$ be such that $\alpha(u)=\sigma\left(u^{2}\right)$ if $u \equiv 2 \bmod 3$ and $\alpha(u)=\sigma\left(u^{2}\right) / 3$ if $u \equiv 1 \bmod 3$. By Lemma $3, \alpha(u)=\alpha(v)$ implies $u=v$. So all primes from $p_{2}$ produce at least two prime factors, except for at most one per distinct prime from $K$. That is, $2 p_{2}-1-p_{2,1}-p_{4} \leq|K|$. Thus, we have $2 p_{2}-1-p_{2,1}-p_{4} \leq e+2 p_{2,1}+f_{4}$, which gives

$$
2 p_{2} \leq 1+e+3 p_{2,1}+p_{4}+f_{4} .
$$

The combination $5 \times(\mathbf{1})+7 \times(\mathbf{2})+5 \times(\underline{\mathbf{3}})+6 \times(\underline{\mathbf{4}})+2 \times(\mathbf{5})+16 \times(\underline{\mathbf{6}})+(\mathbf{7})+2 \times(\underline{\mathbf{8}})$ gives $1 \leq 0$, a contradiction. This means that for assumption (7) that $7 f \leq 18 p-32$ is false, and thus $\Omega(N) \geq(18 \omega(N)-31) / 7$.

\section{Proof of $\Omega(N) \geq 2 \omega(N)+51$}

We use the general method and the computer program discussed in [4].

We use the following contradictions:

- The abundancy of the current number is strictly greater than 2 .

- The current number $n$ satisfies $\Omega(n) \geq 2 \omega(n)+51$.

We forbid the factors in $S=\{3,5,7,11,13,17,19\}$, in this order. We branch on the smallest available prime congruent to $1 \bmod 3$. If there is no such prime, we branch on the smallest available prime congruent to $2 \bmod 3$. We still use a combination of exact branchings and standard branchings, as in [4]. We use exact branchings only for the special components $p^{1}$ and for all the even powers $3^{2 e}$ of 3 .

By-passing roadblocks. A roadblock is a situation such that there is no contradiction and no possibility to branch on a prime. This happens when we have already made suppositions for the multiplicity of all the known primes and the other numbers are composites.

Given a roadblock $M$, we check that the composites involved are not divisible by an already considered prime, are not perfect powers, have no factor less than $10^{10}$, and are pairwise coprime. Then we compute the following quantities:

- $F$ : It is a lower bound on the number of distinct prime factors of $M$. We count the number of known prime factors of $M$ plus two primes per composite number. 
- A: It is an upper bound on the abundancy of $M$. For the abundancy of a component $p^{e}$, we use $\sigma_{-1}\left(p^{e}\right)$ for an exact branching and $\sigma_{-1}\left(p^{\infty}\right)=$ $p /(p-1)$ for a standard branching.

For a composite $C$, we know that $C$ has at most $\left\lfloor\frac{\ln C}{10 \ln 10}\right\rfloor$ prime factors since $C$ has no factor less than $10^{10}$. So, the abundancy due to $C$ is at most $\left(1+10^{-10}\right)^{\left\lfloor\frac{\ln C}{10 \ln 10}\right\rfloor}$.

- $T$ : It is the target lower bound on $\Omega(N)-2 \omega(N)$, thus an odd integer. We use $T=51$ in the proof of Theorem 2 .

For the sake of contradiction, we suppose that $\Omega(N)-2 \omega(N) \leq T-2$. By Theorem 1, we have $\Omega(N) \geq(18 \omega(N)-31) / 7$. So $(18 \omega(N)-31) / 7-2 \omega(N) \leq$ $\Omega(N)-2 \omega(N) \leq T-2$, which gives $\omega(N) \leq(7 T+17) / 4$. Thus, $N$ has at most $\omega(N) \leq(7 T+17) / 4-F$ prime factors that do not divide $M$. Let $p$ be the smallest of these extra factors. We see that if

$$
A(p /(p-1))^{(7 T+17) / 4-F}<2,
$$

then $N$ cannot reach abundancy 2. This gives an upper bound on $p$. To get around the roadblock, we branch on every prime number $p$ (except those that divide $M$ or are already forbidden) in increasing order until (9) is satisfied.

\section{Example.}

$3^{4} \Longrightarrow 11^{2}$

$$
\begin{aligned}
& 11^{18} \Longrightarrow 6115909044841454629 \\
& \quad 6115909044841454629^{16} \Longrightarrow \sigma\left(6115909044841454629^{16}\right) \quad \text { Roadblock } 1 \\
& \quad 5^{1} \Longrightarrow 2 \times 3 \quad \text { Roadblock } 2
\end{aligned}
$$

We first branch on the components $3^{4}, 11^{18}$, and $\sigma\left(11^{18}\right)^{16}$ and hit a first roadblock, as no factors of $C_{1}=\sigma\left(\sigma\left(11^{18}\right)^{16}\right)$ are known. When trying to get around this roadblock, we first branch on $5^{1}$ and hit a second roadblock. Consider this second roadblock:

- $F=6$ : We have the four primes $3,5,11, \sigma\left(11^{18}\right)$, and at least two primes from $C_{1}$.

$$
\begin{aligned}
& \text { - } A=\sigma_{-1}\left(3^{4} \times 5 \times 11^{\infty} \times \sigma\left(11^{18}\right)^{\infty}\right) \times\left(1+10^{-10}\right)^{\left\lfloor\frac{\ln C_{1}}{10 \ln 10}\right\rfloor}=1.9718518 \cdots \text {. } \\
& \text { - } T=51 \text {. }
\end{aligned}
$$

Equation (9) is satisfied for $p \geq 6174$, so to circumvent $M$, we branch on every prime $p$ between 7 and 6173, except 11 .

When $N$ has no factors in $S$. If $N$ has no factor in $S$, then it must have at least 115 distinct prime factors. We obtain this by considering the product $\Pi_{23 \leq p \leq 673} \frac{p}{p-1}=1.99807632 \ldots$ over the first 114 primes $p$ greater than 19 , which is an upper bound on the abundancy and is smaller than 2 .

Using Theorem 1, we obtain

$$
\begin{aligned}
\Omega(N)-2 \omega(N) & \geq(18 \omega(N)-31) / 7-2 \omega(N) \\
& =(4 \omega(N)-31) / 7 \\
& \geq(4 \times 115-31) / 7 \\
& =61+2 / 7 .
\end{aligned}
$$

So, we have $\Omega(N) \geq 2 \omega(N)+62$, which concludes the proof of Theorem 2 , 


\section{ACKNOWLEDGMENT}

We thank Robert Gerbicz for a much simpler proof of Lemma 3 ,

\section{REFERENCES}

[1] Graeme L. Cohen, On the largest component of an odd perfect number, J. Austral. Math. Soc. Ser. A 42 (1987), no. 2, 280-286. MR869751 (87m:11005)

[2] Takeshi Goto and Yasuo Ohno, Odd perfect numbers have a prime factor exceeding $10^{8}$, Math. Comp. 77 (2008), no. 263, 1859-1868, DOI 10.1090/S0025-5718-08-02050-9. MR2398799 (2009b:11008)

[3] Pace P. Nielsen, Odd perfect numbers have at least nine distinct prime factors, Math. Comp. 76 (2007), no. 260, 2109-2126, DOI 10.1090/S0025-5718-07-01990-4. MR2336286 (2008g:11153)

[4] Pascal Ochem and Michaël Rao, Odd perfect numbers are greater than $10^{1500}$, Math. Comp. 81 (2012), no. 279, 1869-1877, DOI 10.1090/S0025-5718-2012-02563-4. MR2904606

[5] Trygve Nagell, Introduction to Number Theory, John Wiley \& Sons Inc., New York, 1951. MR0043111 (13,207b)

[6] R. Steuerwald, Verschärfung einer notwendigen Bedingung für die Existenz einen ungeraden vollkommenen Zahl, S.-B. Bayer. Akad. Wiss. (1937), pp. 69-72.

CNRS, LiRMM, Université Montpellier 2, 161 Rue AdA, 34095 Montpellier Cedex 5, FRANCE

E-mail address: ochem@lirmm.fr

CNRS, LIP, ENS Lyon, 15 Parvis R. Descartes BP 7000, 69342 Lyon Cedex 07, France E-mail address: michael.rao@ens-lyon.fr 